\title{
O Programa Brasileiro de Biodiesel e o Risco Associado ao Preço da Mamona em Irecê, Bahia ${ }^{12}$
}

\author{
Marcelo Dias Paes Ferreira ${ }^{3}$, Lindomar Pegorini Daniel ${ }^{4}$ \\ e João Eustáquio de Lima $^{5}$
}

Resumo: O presente estudo buscou verificar se os preços da mamona no estado da Bahia estão sujeitos à instabilidade estrutural e se a implementação do Programa Nacional de Produção e Uso do Biodiesel (PNPB) elevou tal instabilidade. Foram utilizados modelos TGARCH e EGARCH de heterocedasticidade condicional para medir a instabilidade estrutural dos preços da mamona e o efeito do PNPB sobre a volatilidade dos preços. Os resultados indicam que os preços de mamona na Bahia são instáveis, que aumentos nos preços elevam a instabilidade e que o PNPB aumentou o risco de preço no estado. Dada a aversão ao risco por parte dos produtores, a implementação do PNPB tornou a atividade menos atrativa, o que pode ter contribuído para a baixa relevância da mamona dentro do programa.

Palavras-chaves: Preço ao produtor; PNPB; TGARCH; EGARCH.

Abstract: This study aimed to assess the structural instability of castor bean price in
Bahia state, Brazil, and to verify if The Brazilian National Program for Production and
Use of Biodiesel (PNPB) has increased castor bean price instability. We modeled price
instability by TGARCH and EGARCH conditional heteroscedasticity approaches. Results
point out that castor bean prices in Bahia are structurally volatile, a price increase leads
to a higher instability, and PNPB raised castor bean price volatility in Bahia. As farmers
are risk averse, the PNPB became castor bean cultivation less attractive, which may have
contributed to the null use of castor bean as a raw material within the program.

Key-words: Farm-gate price; PNPB; TGARCH; EGARCH.

DOI - http://dx.doi.org/10.1590/1234-56781806-9479005304006

Classificação JEL: Q18, O13.

1. Data de submissão: 19 de setembro de 2014. Data de aceite: 11 de novembro de 2015.

2. Uma versão preliminar desse trabalho foi apresentada no $51^{\circ}$ Congresso da Sociedade Brasileira de Economia, Administração e Sociologia Rural, realizado em julho de 2013 em Belém (PA).

3. Universidade Federal de Goiás. Goiânia, Goiás, Brasil. E-mail: marcelo.ferreira@ufg.br

4. Universidade Federal de Viçosa, professor da Universidade do Estado do Mato Grosso. Sinop, Mato Grosso, Brasil. E-mail: lindomar.pegorini@gmail.com

5. Universidade Federal de Viçosa. Viçosa, Minas Gerais, Brasil. E-mail: jelima@ufv.br 


\section{Introdução}

O Programa Nacional de Produção e Uso do Biodiesel (PNPB) foi introduzido em 2004 para incentivar o uso do biodiesel em conjunto com o diesel mineral. Ao contrário do Programa Nacional do Álcool (Proácool), associado à monocultura da cana-de-açúcar cultivada em grandes propriedades, o PNPB buscou promover uma base mais diversificada de matérias-primas e a inclusão e o desenvolvimento social de pequenos agricultores (MENDES e COSTA, 2010). Todavia, alguns trabalhos apontam que o PNPB não tem sido totalmente efetivo em inserir produtores que não participavam no mercado de oleaginosas (DINIZ e FAVARETO, 2012; SILVA et al., 2014a).

Uma das matérias-primas que se encaixam na proposta de inclusão social do PNPB é a mamona. Segundo César e Batalha (2011), o governo federal escolheu essa cultura como estratégica no contexto do PNPB, uma vez que apresenta baixo custo de implantação e produção, adequação às condições climáticas do semiárido, sendo apta a ser produzida em grande parte do Nordeste brasileiro. Ademais, esses autores indicaram que a mamona é uma cultura mão de obra intensiva, o que implica maior geração de renda dado o maior nível de emprego rural temporário. Assim, considerando o fato de o Nordeste concentrar boa parte da pobreza rural no Brasil e ser uma área de clima semiárido, a mamona seria ideal para o cumprimento dos objetivos do PNPB (SCHOLZ e SILVA, 2008). Contudo, o interesse do governo federal pela mamona não se traduziu em números efetivos, uma vez que dados da Agência Nacional do Petróleo (ANP, 2015) indicam que mais de $80 \%$ da matéria-prima utilizada para a produção do biodiesel é óleo de soja, enquanto a parcela referente à mamona é nula. Contribui para essa parcela nula o fato de o óleo de mamona apresentar diversos usos na indústria química fina, o que não torna a mamona uma alternativa custo-efetiva para a produção de biodiesel (GONÇALVES, FAVARETO e ABRAMOVAY, 2013). Todavia, a entrada de empresas de biodiesel como possíveis demandantes de mamona aumentou a concorrência pela matéria-prima e elevou os preços pagos aos produtores (DINIZ e FAVARETO, 2012; GONÇALVES, FAVARETO e ABRAMOVAY, 2013).

O principal estado produtor de mamona no Brasil é a Bahia. Ao longo da última década, esse estado concentrou cerca de $80 \%$ da produção nacional, especialmente na região de Irecê (IBGE, 2015). A produção na Bahia parece não ser influenciada pela elevação de preços no âmbito do PNPB, apresentando o seguinte padrão da produção e do preço ao longo dos anos (Figura 1): a) de 2001/02 a 2003/04, tanto o preço quanto a quantidade produzida apresentaram incrementos; b) em 2004/05, ocorreu aumento na produção e queda no preço; c) em 2005/06, ambos os indicadores caíram; d) nos dois anos seguintes, 
ambos variaram positivamente e e) a partir de então, os preços apresentaram uma tendência de alta, enquanto a produção apresentou tendência de queda. Considerando todo o período, constata-se que a produção caiu a uma taxa de $1 \%$ ao ano, enquanto o preço aumentou a uma taxa de $2,6 \%$ ao ano. O PNPB não elevou a produção de mamona no estado da Bahia, haja vista que o nível de produção atual está abaixo do observado no início dos anos 2000. Apesar de a produção de mamona não ser exclusivamente determinada pelos preços, o que se observa é que não houve incremento na produção associado aos cenários de incentivo público e aumento de preços.

Há diversos elementos que impendem o aumento da oferta de mamona na Bahia, tais como a oferta de matéria-prima pulverizada e organizada em bases tradicionais e a instabilidade de preços (GONÇALVES, FAVARETO e ABRAMOVAY, 2013). Esse último elemento pode ter influenciado negativamente a produção dada a aversão ao risco por parte produtores familiares. A Figura 1 mostra que a produção e o preço da mamona na Bahia variaram de maneira significativa ao longo da última década. O coeficiente de variação da produção situa-se em $36 \%$ e o do preço, em torno de $23 \%$ no período. O coeficiente de variação para preços mundiais de alimentos foi de $18 \%$ para o mesmo período (FAO, 2012), indicando maior instabilidade relativa para o mercado da mamona. Segundo Todaro e Smith (2012), pequenos agricultores são mais avessos a riscos, uma vez que isso traria consequências para a sobrevivência da família. Os pequenos produtores também têm menor acesso a crédito e assistência técnica. No entanto, mesmo quando têm acesso por meio de programas de crédito, assistência técnica ou de preços mínimos, a aversão ao risco os impede de incrementar, inovar ou migrar para culturas ou técnicas que apresentam maior nível médio de produtividade ou retorno, caso elas também apresentem maior variância (risco/volatilidade) associada ao preço ou à produtividade (TODARO e SMITH, 2012). Esse fenômeno estaria relacionado à segurança alimentar da família, ou seja, os produtores têm preferência por culturas ou sistemas de produção que lhes proporcionem maior segurança/menor risco. Especificamente no caso da mamona, Viana, Perez e Machado (2011) constataram que a produção de mamona na região de Irecê apresenta alto risco. Em um dos cenários analisados, esses autores verificaram que a chance de um agricultor apresentar desempenho econômico negativos é de 70\%. Assim, qualquer aumento na instabilidade de preços pode tornar a atividade menos atrativa, aumentando a probabilidade de prejuízo.

Figura 1. Evolução indexada da produção de mamona e do preço pago ao produtor no estado da Bahia

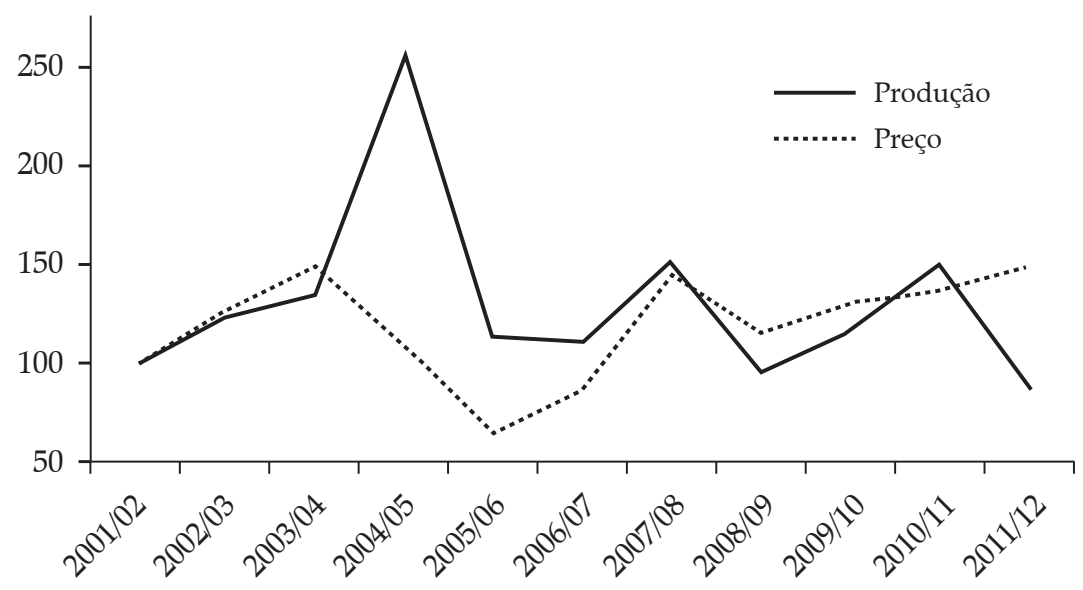

Fonte: Conab (2013) e Seagri (2013). 
Nesse sentido, se os preços da mamona são voláteis (i.e. possuem um componente de risco estrutural), o aumento de preço por si só não incentivaria a produção, mesmo no contexto dos incentivos promovidos pelo PNPB. O aumento do preço pago pela mamona também pode ter influenciado a volatilidade, uma vez que para alguns produtos agrícolas no Brasil há uma relação entre aumentos de preços e aumento de volatilidade (SILVA, SÁFADI e CASTRO JÚNIOR, 2005). Ademais, por se caracterizar como uma inovação no mercado de mamona, a própria implantação do PNPB pode ter alterado a estrutura do mercado de mamona e, por conseguinte, a volatilidade dos preços na Bahia. Esse aumento de instabilidade atribuído ao PNPB poderia se dar de diversas formas. Por exemplo, alguns autores indicam que, no início do programa, houve um movimento de entrada de usinas biodiesel que posteriormente deixaram a região produtora de mamona (CÉSAR e BATALHA, 2010a, 2010b, 2011). Outro fator que pode ter aumentado a instabilidade é o aumento da influência de variações em mercados de outros óleos vegetais substitutos tais como o óleo de soja, o que não ocorria anteriormente dada a especificidade do uso do óleo de mamona na indústria química. ${ }^{6}$

Portanto, o presente estudo se propõe a analisar a volatilidade estrutural dos preços da mamona na Bahia e se essa volatilidade apresentou incrementos no período pós-PNPB. Diante do exposto, o objetivo geral desse trabalho é verificar se há riscos associados a preços pagos aos produtores de mamona na Bahia. Especificamente, pretendeu-se: a) apresentar a sistemática do risco do preço da mamona; $b$ ) verificar se preços mais altos elevam o risco de mercado e c) contrastar o risco de preço antes e depois do PNPB. Diante dos objetivos propostos, este trabalho contribui na avaliação do PNPB trazendo uma perspectiva quantitativa, complementando os trabalhos de caráter qualitativos desenvolvidos até agora. De forma mais específica, este estudo provê uma

6. A seção número 2 apresenta de forma detalhada os principais fatos que ocorreram ao longo do PNPB e que podem ter contribuído para o aumento da volatilidade de preços. dimensão quantitativa da instabilidade no mercado da mamona verificada em diversos trabalhos qualitativos (CÉSAR e BATALHA, 2010a, 2010b, 2011; DINIZ e FAVARETO, 2012; GONÇALVES, FAVARETO e ABRAMOVAY, 2013).

Este artigo está organizado em mais quatro seções. Na próxima seção consta uma breve revisão de literatura em que são apresentados os principais resultados para análises do complexo mamona no Brasil e a implicação do PNPB na instabilidade de preços da mamona no estado da Bahia. A seção 3 apresenta a metodologia aplicada para alcançar os objetivos propostos, elucidando o método estatístico empregado e a seleção e o tratamento das variáveis. O procedimento consiste na estimação dos modelos TGARCH e EGARCH de heterocedasticidade condicional. A seção 4 apresenta os resultados e a discussão do trabalho, observando as características relevantes do risco associado ao preço da mamona. Por fim, a seção 5 apresenta as considerações finais.

\section{O PNPB e a produção de mamona}

O PNPB se destaca de programas de outros países pelo fato de visar o desenvolvimento socioeconômico relacionado à inserção de agricultura familiar nessa cadeia de produção, contudo, tal inclusão não tem ocorrido no caso da mamona (CÉSAR e BATALHA, 2010a, 2010b). As dificuldades apontadas para inserção da mamona como matéria-prima para a produção de biodiesel na Bahia são: a utilização de técnicas inadequadas, assistência técnica deficiente, condições edafoclimáticas desfavoráveis, oferta pulverizada, atravessadores na comercialização, reduzida capacidade de investimento do agricultor familiar, quebra de contratos por parte das usinas, aversão à participação em cooperativas por parte dos produtores e agricultores endividados (CAMPOS et al., 2009; CÉSAR e BATALHA, 2010b; DINIZ e FAVARETO, 2012; GONÇALVES, FAVARETO e ABRAMOVAY, 2013).

A estratégia de inclusão de pequenos agricultores na base produtiva do PNPB se deu por meio 
da criação do selo combustível social, certificação gerida pelo Ministério do Desenvolvimento Agrário. Para conseguir o selo, a usina deve comprar uma quantidade mínima da matéria-prima proveniente de agricultores ou cooperativas que possuem declaração de aptidão ao Programa Nacional de Fortalecimento da Agricultura Familiar, fornecer serviços de assistência técnica e desenvolver programas de capacitação. Atualmente, o percentual mínimo exigido de matéria-prima da agricultura familiar é de 30\% na região Nordeste (SILVA et al., 2014b). As vantagens para as usinas certificadas são a isenção parcial de contribuições como PIS/Pasep e Cofins, incentivos comerciais e financeiros e a participação dos leilóes referentes a $80 \%$ do volume de biodiesel comercializado (DINIZ e FAVARETO, 2012; SILVA et al., 2014b).

$\mathrm{O}$ ambiente institucional e o não cumprimento dos contratos têm contribuído sobremaneira para a instabilidade do setor. Empresas que compram mamona na Bahia para obter o selo combustível social incorrem em altos custos de transação relativos à gestão da comercialização com um número elevado de agricultores familiares. O não cumprimento dos contratos por parte das empresas de biodiesel no que concerne à compra e à provisão de assistência técnica fez com que os produtores de mamona perdessem a confiança nas empresas, tendo aumentado a aversão à participação no PNPB (CÉSAR e BATALHA, 2010b).

César e Batalha (2010a, 2010b) indicaram que empresas que inicialmente se instalaram na região Nordeste para produzir biodiesel encerraram suas atividades ou se deslocaram para outras regiões produtoras de outras oleaginosas onde a atividade seria mais viável. Os incentivos fiscais dados à produção de biodiesel proveniente da matéria-prima familiar não eram suficientes para cobrir os custos relacionados à coordenação da cadeia de mamona, tornando o biodiesel produzido no Nordeste menos competitivo em relação ao produzido nas regiões Sul e Centro-Oeste (CÉSAR e BATALHA, 2010a). Apenas três empresas de biodiesel compravam mamona no
Nnordeste entre 2008 e 2009, e o faziam exclusivamente para obter o selo combustível social (CÉSAR e BATALHA, 2010b, 2011).

A desistência por parte das usinas também está associada à competição por matéria-prima com a indústria ricinoquímica. A entrada de usinas de biodiesel forçou a demanda por mamona, retirando matéria-prima do mercado. Os derivados do óleo de mamona usados na indústria química possuem aplicações específicas, o que os tornam relativamente caros. Assim, a indústria ricinoquímica elevou o preço pago pela saca de mamona, pois tinham margem para aumentar seus custos sem incorrer em prejuízos (DINIZ e FAVARETO, 2012; GONÇALVES, FAVARETO e ABRAMOVAY, 2013). Todavia, o novo nível de preços elevou os custos de produção de biodiesel de mamona para um patamar economicamente inviável.

Outro aspecto que pode ter promovido a desistência por parte de algumas usinas em investir na base produtiva de mamona está associado a questões químicas. O óleo de mamona possui características que o tornam mais viscoso que os demais óleos vegetais, o que inviabiliza a produção de biodiesel unicamente de mamona (MENDES e COSTA, 2010). Dessa forma, além de agir como coordenadora da cadeia de mamona para produção do biodiesel, a usina também deveria atuar em mercados de outras oleaginosas, aumentando seus custos de transação.

Problemas na produção da mamona também foram observados em outras regiões brasileiras no contexto do PNPB. Watanabe, Bijman e Slingerland (2012) compararam as relações dos produtores familiares de mamona e soja que forneciam matéria-prima para uma usina de biodiesel no norte de Minas Gerais. Enquanto a usina negocia com a cooperativa dos produtores de soja, o que reduz seus custos de transação, a negociação com os produtores de mamona é feita de maneira mais dispersa. Ademais, os agricultores de mamona geralmente produzem culturas alimentares, destinando apenas uma pequena parte das áreas para mamona a fim de assumir um risco alimentar menor. Os produtores de soja encaram um menor custo de transação no que 
concerne à opção de venda do produto, uma que vez que podem oferecer o produto tanto no mercado convencional, quanto no de biodiesel. Os produtores de mamona no norte de Minas Gerais, entretanto, não têm um mercado alternativo, o que gera dependência por parte dos produtores, elevando seus custos de transação. Tais custos de transação observados tanto pelas usinas quanto pelos agricultores familiares poderiam estar afetando a produção de mamona na Bahia, haja vista que a realidade do norte de Minas Gerais e do semiárido baiano é semelhante.

A conjuntura descrita nos trabalhos citados (CÉSAR e BATALHA, 2010a, 2010b, 2011; DINIZ e FAVARETO, 2012; GONÇALVES, FAVARETO e ABRAMOVAY, 2013; MENDES e COSTA, 2010; SILVA et al., 2014b; WATANABE, BIJMAN e SLINGERLAND, 2012) permite constatar certa instabilidade no mercado de mamona na Bahia. A entrada de novos agentes nesse mercado, na forma das usinas de biodiesel, levou a uma mudança na competição por compra de mamona, elevando o preço praticado no mercado. Todavia, os problemas estruturais apresentados fizeram com que as usinas de biodiesel abandonassem seus projetos na região Nordeste, trazendo mais instabilidade para o setor. Tal instabilidade pode ser traduzida pelo aumento da volatilidade dos preços da mamona, que estaria associada ao aumento do risco de preço para a cultura.

A inserção da mamona ao PNPB traz outro aspecto que pode aumentar a instabilidade nos preços. Pelo fato de a base de matérias-primas para a produção de biodiesel ser diversificada (óleo de soja, óleo de algodão, óleo de dendê, gorduras, animais etc.), choques que ocorreriam nos mercados dessas matérias-primas poderiam afetar o mercado de mamona, haja vista que são produtos substitutos para a produção de biodiesel.

$\mathrm{O}$ aumento na instabilidade do preço pode ter consequências negativas sobre a decisão do agricultor familiar em plantar mamona, mesmo em um contexto de aumento de preços. Pelo fato de tal agricultor não utilizar técnicas modernas e encarar elevada restrição de capital, ele não estaria propenso a plantar mamona em detrimento das culturas alimentares, sabendo que aceitaria um risco maior de não vender a produção por um preço favorável em um contexto de maior volatilidade. É fato que os mercados dos produtos alimentares no Brasil também são voláteis (SAITH e KAMITANI, 2012; SILVA, SÁFADI e CASTRO JÚNIOR, 2005), todavia, na pior das hipóteses, o agricultor poderia se alimentar da produção, ou alimentar seus animais, o que não é factível para a mamona pelo fato de ser uma planta tóxica.

\section{Metodologia}

A série utilizada neste estudo refere-se aos preços recebidos pelos produtores pela saca de $60 \mathrm{~kg}$ da mamona em baga na Bahia, praça de Irecê, oriundos da Secretaria de Agricultura da Bahia (Seagri). Os dados são de frequência semanal, abrangendo o período de 09/01/2002 a 29/06/2011, totalizando 488 observações. A série de preços não foi deflacionada por dois motivos: frequência semanal dos dados e, principalmente, pelo fato de se trabalhar com a série de retornos, cujo componente de longo prazo, no caso a inflação, deixa de exercer influência significativa. Para analisar a volatilidade e a assimetria dos preços da mamona na Bahia, foi utilizado o retorno dos preços, definido como:

$$
r_{\mathrm{t}}=\ln P_{\mathrm{t}}-\ln P_{\mathrm{t}-1}
$$

em que $r_{\mathrm{t}}$ é o retorno do preço da mamona na semana $t ; \ln P_{\mathrm{t}}$ é o logaritmo do preço da mamona na semana $t$; e ln $P_{\mathrm{t}-1}$ é o logaritmo do preço da mamona na semana $t-1$.

Algumas das vantagens em adotar a taxa de retorno para a análise da série de preços é o fato de as séries de retornos serem geralmente estacionárias em nível e, segundo a teoria do portfólio, ser possível relacionar o retorno esperado da atividade com o risco/volatilidade associado a ela.

Para a análise da volatilidade associada à série de retornos dos preços da mamona na Bahia, foram consideradas as informações de estatísticas descritivas como a média, desvio padrão, assimetria e curtose, que fornecem evidências sobre risco e retorno 
esperado e sobre a distribuição incondicional dos retornos. Para testar se o processo seguia a distribuição normal, foi utilizado o teste de normalidade de Jarque-Bera. E mesmo as séries de retornos tendo comportamento estacionário, aplicou-se o tradicional teste ADF (Dickey-Fuller Aumentado) (DICKEY e FULLER, 1979, 1981), para avaliar formalmente as condições de estacionariedade da série.

Como o objeto de interesse do presente estudo é a volatilidade, é natural que o procedimento empírico adotado seja a estimação de algum modelo da família ARCH/GARCH. Esses modelos se concentram em técnicas que permitem a modelagem temporal de variâncias e covariâncias, ou seja, eles se fundamentam na estimação da variância condicional, em lugar de considerá-la constante ao longo do tempo. Para tanto, é necessário especificar um modelo para a média condicional que elimine a possível autocorrelação existente. Este modelo é do tipo autorregressivo e de médias móveis ARMA, primeiramente exposto por Whitle (1951), sendo expresso por:

$$
r_{t}=c+\sum_{i=1}^{k} \phi_{i} r_{t-1}+\varepsilon_{t}+\sum_{i=1}^{s} \theta_{i} \varepsilon_{t-s}
$$

em que $r_{\mathrm{t}}$ é o retorno do preço da mamona; $\phi_{\mathrm{i}} r_{\mathrm{t}-\mathrm{i}}$ são os componentes autorregressivos do modelo; $\theta_{\mathrm{i}} \varepsilon_{\mathrm{t}-\mathrm{s}}$ são os componentes das médias móveis; e $\varepsilon_{\mathrm{t}}$ é o termo de erro com as propriedades desejáveis. Como geralmente os retornos não são autocorrelacionados, espera-se que o modelo para a média condicional seja estimado apenas com a constante, conhecido como modelo de Retorno Esperado Constante (CER) pela teria do portfólio.

Por fim, busca-se identificar a existência de heterocedasticidade na variância condicional, ou seja, evidenciar a presença do efeito $\mathrm{ARCH} /$ GARCH na série de retornos dos preços da mamona e modelar tal efeito. Evidências para a existência de efeito ARCH/GARCH podem ser obtidas pelos gráficos dos retornos e dos retornos ao quadrado. A ocorrência de clusters de volatilidade indica que os retornos não são independentes e identicamente distribuídos (iid) e, portanto, que existe heterocedasticidade condicional. E as funções de autocorrelação e autocorrelação par- cial dos resíduos ao quadrado da equação da média podem sugerir variância não constante, assim como o teste do tipo Multiplicador de Lagrange proposto por Engle (1982), cuja estatística de teste apresenta distribuição qui-quadrado:

$$
\varepsilon_{t}^{2}=\beta_{0}+\sum_{i=1}^{q} \beta_{i} \varepsilon_{t-i}^{2}+v_{t}
$$

A hipótese nula de variância condicional constante é testada comparando os valores calculado e tabelado. Ademais, para atender aos objetivos propostos pelo estudo foram utilizados o TGARCH e o EGARCH, dois dos modelos de heterocedasticidade condicional.

Os modelos de heterocedasticidade condicional se baseiam numa especificação autorregressiva para a variância, constituindo os modelos $\mathrm{ARCH}$, GARCH, TGARCH e EGARCH diferentes formas de especificação para a variância condicional. ${ }^{7}$

Apesar de mais parcimoniosos, os modelos ARCH e GARCH consideram que os impactos dos choques aleatórios são simétricos. Para superar essa limitação, desenvolveu-se o modelo TGARCH (heterocedasticidade condicional autorregressiva generalizado com limiar), introduzido independentemente por Glosten, Jaganathan e Runkle (1993) e Zakoian (1994). A forma funcional do modelo TGARCH considera a variância função dos termos de erro ao quadrado defasados da equação da média e de valores defasados da variância condicional:

$$
\begin{aligned}
\sigma_{t}^{a}= & \alpha_{0}+\sum_{i=1}^{q} \alpha_{i} \varepsilon_{t-1}^{a}+\sum_{j=1}^{p} \beta_{j} \sigma_{t-j}^{a}+ \\
& +\sum_{k=1}^{r} \gamma_{k} d_{\left(\varepsilon_{-l-} \leq 0\right)}\left|\varepsilon_{t-k}\right|^{j a}
\end{aligned}
$$

em que $q$ e $p$ representam a ordem de defasagem dos termos de erro quadráticos e da variância, respectivamente, e indicam a ordem do modelo TGARCH; e $d$ é uma variável dicotômica que assume valor 1 se o choque for negativo $\left(\varepsilon_{t-k} \leq 0\right)$. $\mathrm{O}$ coeficiente $a$ indica o expoente da variância e, apesar de normalmente tomar o valor 2, ele é livre.

7. Maiores detalhes sobre os modelos da família ARCH/ GARCH podem ser encontrados em Bollerslev (1986), Bueno (2008), Engle (1982) e Taylor (1986) 
No modelo TGARCH, é possível verificar a assimetria que ocorre sobre a volatilidade quando há choques positivos ou negativos. O efeito de choques positivos sobre variância condicional é representado pelo coeficiente $\alpha_{\mathrm{i}}$, já o efeito de choques negativos é representado por $\left(\alpha_{i}+\gamma_{k}\right)$. Dessa forma, dado $\alpha_{i}>0$ e definida a defasagem, os choques negativos exercerão maior efeito que choques positivos sobre a volatilidade caso $\gamma_{k}>0$, menor efeito caso $\gamma_{k}<0$ e o mesmo efeito (simétricos) caso $\gamma_{\mathrm{k}}=0$ (BUENO, 2008).

Uma forma alternativa para captar assimetria entre choques negativos e positivos na volatilidade é fornecida pelo modelo EGARCH (heterocedasticidade condicional autorregressivo generalizado exponencial) proposto por Nelson (1991). Além disso, o EGARH permite que alguns parâmetros sejam negativos. Novamente a variância condicional é função dos termos de erro defasados da equação da média e de termos autorregressivos da variância condicional, porém, considerando outra forma funcional:

$$
\begin{aligned}
\ln \left(\sigma_{t}^{2}\right)= & \alpha_{0}+\sum_{j=1}^{p} \beta_{j} \ln \left(\sigma_{t-j}^{2}\right)+\sum_{i=1}^{q} \alpha_{i}\left|\frac{\varepsilon_{t-i}}{\sigma_{t-i}}\right|+ \\
& +\sum_{k=1}^{r} \alpha_{k} \frac{\varepsilon_{t-k}}{\sigma_{t-k}}
\end{aligned}
$$

Sua forma logarítmica impede que a variância seja negativa, possibilitando que os coeficientes sejam negativos. Os parâmetros $\alpha_{\mathrm{k}}$ captam a presença de assimetria, se $\alpha_{k}=0$, para todo $k$, então não ocorrem efeitos assimétricos, caso contrário, se $\alpha_{k} \neq 0$, para algum $k$, então os impactos serão assimétricos. Se $\alpha_{k}<0$, então choques negativos aumentam mais a volatilidade que choques positivos (BUENO, 2008). No caso do modelo EGARCH, o coeficiente $\beta_{\mathrm{j}}$ indica a persistência de choques sobre a volatilidade.

Os modelos TGARCH e EGARCH, por captarem assimetria, são estimados para analisar a volatilidade dos retornos dos preços da mamona. Adicionalmente, incluiu-se uma variável categórica com valor 0 no período 2002-2003 e valor 1 entre 2004-2011 com o intuito de captar diferenças de risco no preço no período pós-PNPB, em linha com o modelo utilizado por Otuki, Weydmann e Seabra (2009). O coeficiente dessa variável categórica indica se a implantação do PNPB teve efeito elevação (se estatisticamente maior que zero), redução (se estatisticamente menor que zero) ou nulo (se não significativo) sobre o nível de volatilidade do preço da mamona no estado da Bahia. Tal variável mediria o efeito do PNPB sobre o risco de preço da mamona na Bahia.

\section{Resultados e discussão}

\subsection{Análise da série de retornos dos preços da mamona na Bahia}

As Figuras 2 e 3 mostram a série semanal de preços e de retornos dos preços da mamona, respectivamente, para o período de janeiro de 2002 a junho de 2011. Observa-se, na Figura 2, que nos dois primeiros anos da análise houve uma tendência de aumento nos preços da mamona na Bahia. Após um pequeno período de preços baixos observados no final de 2003, os preços da mamona apresentaram um expressivo aumento ao longo de 2004, consequência da introdução do PNPB. O boom no setor com o advento do PNPB levou a um aumento na produção nos anos subsequentes. $\mathrm{O}$ aumento na produção, associado à saída de importantes usinas de biodiesel do mercado da mamona, levou a um cenário de queda generalizada nos preços. Os preços permaneceram em patamares baixos em 2005, 2006 e 2007. Com a queda na produção e o aumento da compra de mamona por parte de algumas usinas de biodiesel, para a obtenção do selo combustível social, houve aumento nos preços, que se mantiveram em patamares relativamente elevados a partir do final de 2007 até o final da amostra. O expressivo aumento observado nos últimos meses de 2011 foi decorrente do fortalecimento da demanda de mamona no mercado brasileiro e da seca que assolou a região produtora da Bahia a partir do final de 2011. 
Figura 2. Série dos preços semanais de mamona pagos ao produtor no estado da Bahia entre janeiro de 2002 e junho de 2011

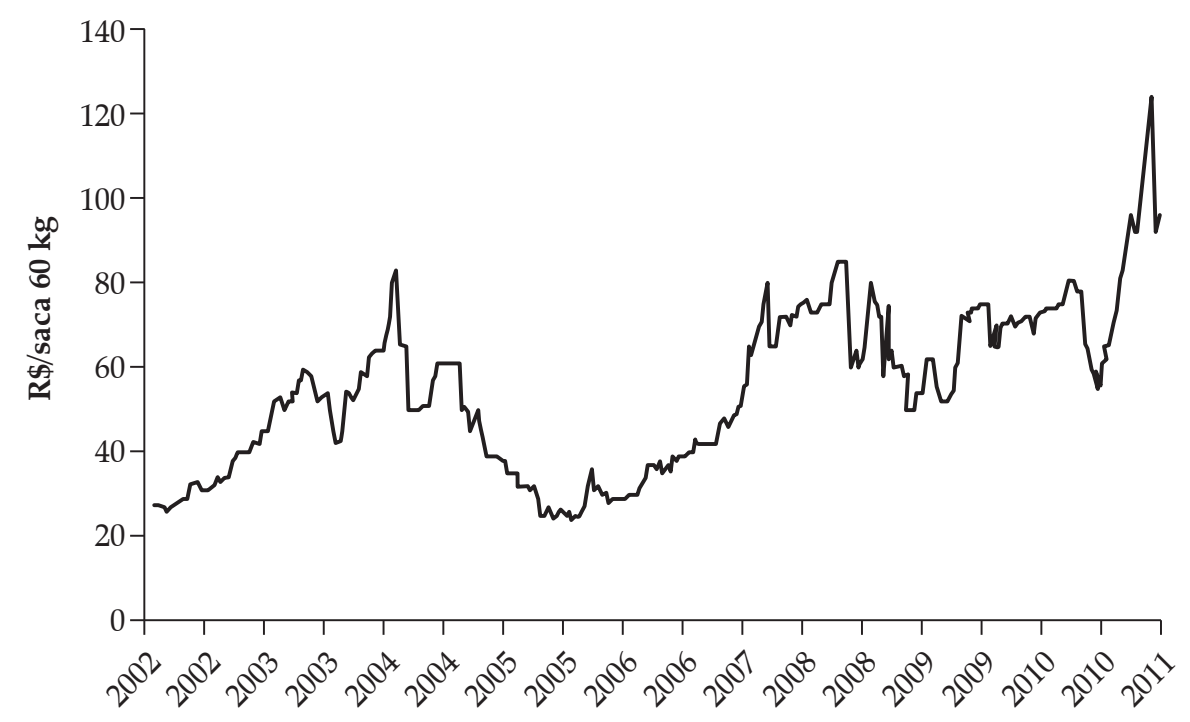

Fonte: Seagri (2013).

Figura 3. Série de retornos dos preços semanais de mamona pagos ao produtor no estado da Bahia entre janeiro de 2002 e junho de 2011

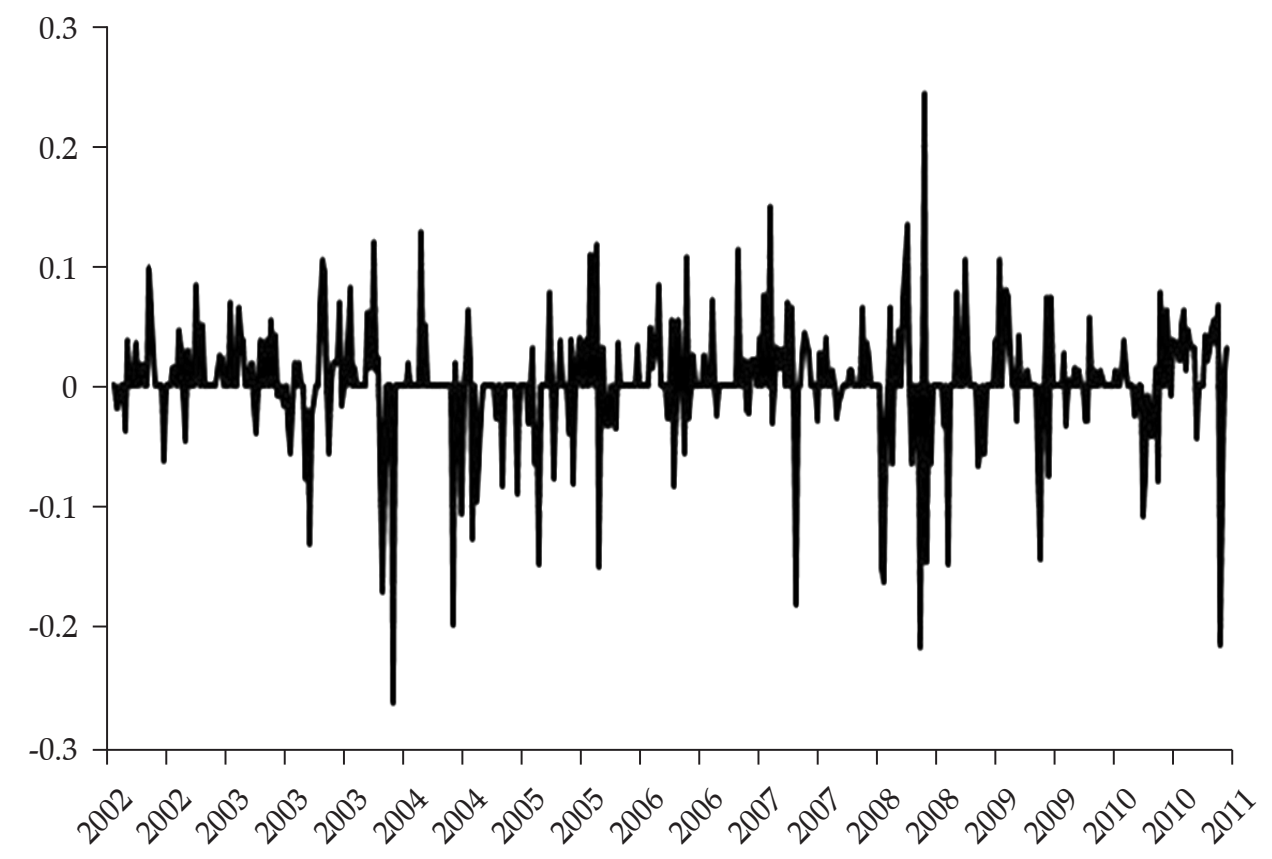

Fonte: Resultados da pesquisa. 
A Figura 3 mostra que o preço apresentou alta volatilidade no período, mais expressiva nos últimos anos. A variabilidade dos preços da mamona no período pode ser atribuída à variação na produção (Figura 1), às políticas de incentivo à produção empreendidas pelo PNPB, ao estabelecimento e ao abandono de empresas na região e a choques oriundos de mercados de outras oleaginosas.

A Figura 3 mostra três pontos de maior instabilidade nos retornos do preço da mamona: em 2004, imediatamente após a implantação do PNPB; no período entre o fim de 2008 e início de 2009 , que coincide com a crise financeira internacional; e, por último, em meados de 2011, quando ocorreu um novo pico de volatilidade associado à seca na região Nordeste. Além disso, é possível observar que a série de retornos tem reversão para a média e, portanto, parece ser estacionária em torno da média zero.

Os clusters de volatilidade podem ser mais claramente visualizados na Figura 4, que apresenta o quadrado dos retornos dos preços da mamona. A ocorrência de três agrupamentos de volatilidade, a exemplo da Figura 3, indica que os retornos não são independentes e identicamente distribuídos (iid), havendo heterocedasticidade condicional na série de retornos da mamona.

A Tabela 1 mostra estatísticas descritivas da distribuição incondicional dos retornos da mamona. A média indica que o retorno esperado para o período é positivo, no entanto, é muito próximo de zero, e associado a um desvio padrão relativamente alto, gera um coeficiente de variação de $1.868 \%$, evidenciando alta volatilidade/risco relacionado à série de retornos. Os coeficientes de assimetria e curtose indicam uma distribuição com assimetria negativa e achatamento caudal, ou seja, a distribuição apresenta caudas com maior massa de probabilidade, que indicam maior ocorrência de eventos extremos e que os retornos seguem uma distribuição leptocúrtica, características de séries que apresentam heterocedasticidade condicional. $\mathrm{O}$ alto valor da estatística do teste Jarque-Bera confirma a não normalidade da distribuição incondicional dos retornos da mamona. As estatísticas descritivas também sinalizam a presença de heterocedasticidade e blocos de volatilidade.

Figura 4. Série de quadrados dos retornos dos preços semanais de mamona pagos ao produtor no estado da Bahia entre janeiro de 2002 e junho de 2011

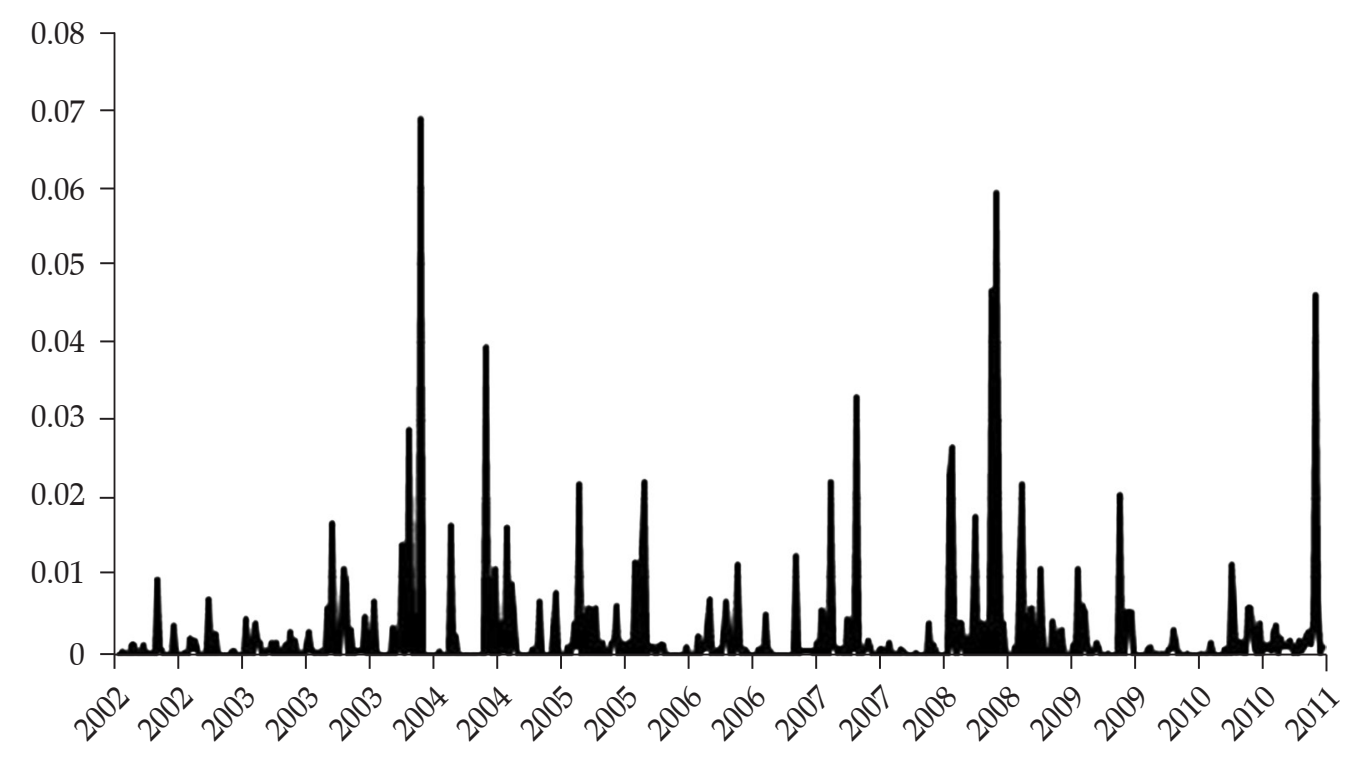

Fonte: Resultados da pesquisa. 
Tabela 1. Estatísticas descritivas para os retornos do preço da mamona

\begin{tabular}{lc}
\hline \multicolumn{1}{c}{ Estatísticas } & Retorno Mamona \\
\hline Média & 0,002 \\
Desvio padrão & 0,047 \\
Coeficiente de variação & 1.868 \\
Assimetria & $-1,051$ \\
Curtose & 9,823 \\
Jarque-Bera & 1.034 \\
\hline
\end{tabular}

Fonte: Resultados da pesquisa.

A Tabela 2 apresenta os resultados do teste ADF para presença de raiz unitária. A conclusão é de que a hipótese nula de presença de raiz unitária pode ser rejeitada ao nível de $1 \%$ de significância, apresentando evidência de que a série é estacionária em nível.

Verificada a estacionariedade da série dos retornos dos preços da mamona, o próximo passo é a identificação da equação para a média e a confirmação da presença de heterocedasticidade/efeito ARCH nos seus resíduos. As funções de autocorrelação (FAC) e autocorrelação parcial (FACP) da série de retornos apontam ausência de autocorrelação, corroborada pelo teste do tipo Multiplicador de Lagrange proposto por Breusch e Godfrey (1981) (Tabela 3).

Pelo teste de Breusch e Godfrey, é possível confirmar ausência de autocorrelação na série de retornos. Nesse caso, estima-se uma equação para a média condicional apenas com a constante como variável explicativa (modelo CER). Após estimar o modelo CER e dadas as evidências anteriores de presença de heterocedasticidade, a FAC e a FACP do quadrado dos resíduos do modelo CER apresentaram defasagens significativas, indicando presença de heterocedasticidade condicional na série de retornos, confirmada pelo teste de Engle (1982) para presença de efeito ARCH (Tabela 4). O teste de Engle (1982) confirma, portanto, que a série de retornos dos preços de mamona na Bahia apresenta variância condicional não constante relevante. Nesse caso é necessário modelar e estimar uma equação para a heterocedasticidade condicional.

Tabela 2. Resultados do teste Dickey-Fuller Aumetado - $\mathrm{ADF}^{*}$

\begin{tabular}{lcc}
\hline \multicolumn{1}{c}{ Teste de Raiz Unitária } & Estatística-t & Probabilidade \\
\hline H0: Retorno da mamona tem raiz unitária & & \\
\hline Estatística de teste ADF & $\mathbf{- 2 0 , 8 3 0 9}$ & $\mathbf{0 . 0 0 0 0}$ \\
\hline Valor crítico a 1\% & $-3,9770$ & \\
Valor crítico a 5\% & $-3,4190$ & \\
Valor crítico a 10\% & $-3,1321$ & \\
\hline
\end{tabular}

* Equação de teste incluindo intercepto e tendência.

Fonte: Resultados da pesquisa.

Tabela 3. Resultados do teste de autocorrelação serial

\begin{tabular}{lcc}
\multicolumn{1}{c}{ Teste de autocorrelação serial } & Estatística & Probabilidade \\
\hline H0: Ausência de autocorrelação & & \\
\hline Estatística de teste F & 0,7778 & 0,6503 \\
Estatística de teste $\left(\right.$ Observações $\left.{ }^{*} \mathrm{R}^{2}\right)$ & 7,8300 & 0,6454 \\
\hline
\end{tabular}

Fonte: Resultados da pesquisa.

Tabela 4. Resultados do teste para efeito ARCH: Engle (1982)

\begin{tabular}{lcc}
\hline \multicolumn{1}{c}{ Teste de autocorrelação serial } & Estatística & Probabilidade \\
\hline H0: Homocedasticidade & & \\
\hline Estatística de teste $\mathrm{F}$ & 2,4680 & 0,0858 \\
Estatística de teste $\left(\right.$ Observações $\left.{ }^{*} \mathrm{R}^{2}\right)$ & 4,9164 & 0,0855 \\
\hline
\end{tabular}

Fonte: Resultados da pesquisa. 


\subsection{Análise da volatilidade/risco da série de} retornos dos preços da mamona na Bahia

Para atender aos objetivos do presente estudo, foram estimadas algumas variações dos modelos de heterocedasticidade condicional que captam assimetria nos choques/inovações TGARCH e EGARCH. Para seleção do modelo mais representativo foram usados os critérios de Akaike (AIC) e Schwarz (SBC) e do Logaritmo da Função de Verossimilhança. As Tabelas 5 e 6 apresentam os modelos estimados e as estatísticas relacionadas.

Em ambos os modelos, a constante para a equação da média foi não significativa. No entanto, os coeficientes associados aos efeitos $\mathrm{ARCH}$ e GARCH foram todos relevantes para explicar a variância condicional. Os resultados indicam que os modelos TGARCH $(2,2)$ e EGARCH $(2,2)$ são os que apresentam melhor desempenho relativo para análise da volatilidade da série de retornos da mamona. Testes para efeito ARCH pós-estimação dos modelos indicam homocedasticidade nos resíduos, ratificando a validade dos resultados.
Quanto à sistemática do risco do preço da mamona, observou-se que os coeficientes de persistência do modelo TGARCH $(2,2)$, considerando choques simétricos, somam -0.052549 para a primeira defasagem e 0.886089 para a segunda defasagem (Tabela 5).

Resultados semelhantes foram encontrados no modelo EGARCH $(2,2)$ em que, considerando novamente choques simétricos, os coeficientes de persistência foram -0.126091 e 0.883247 para a primeira e segunda defasagens, respectivamente (Tabela 6). Esse fato sinaliza que choques de uma semana anterior se dissipam rapidamente, não influenciando de forma relevante a volatilidade dos preços. Todavia, choques defasados em duas semanas instabilizam os preços de forma significativa. Dessa forma, há um hiato de duas semanas entre o choque e seu efeito sobre a variância dos preços nas duas abordagens estimadas. Constata-se, dessa forma, que há volatilidade estrutural nos preços da mamona no estado da Bahia. Assim, choques nos preços tornam o mercado da mamona mais suscetível a variações

Tabela 5. Comparação de desempenho entre os modelos TGARCH de volatilidade

\begin{tabular}{|c|c|c|c|c|}
\hline Retornos Mamona & TGARCH $(\mathbf{1}, \mathbf{1})$ & TGARCH $(2,1)$ & TGARCH $(1,2)$ & TGARCH $(2,2)$ \\
\hline Equação da média & & & & + \\
\hline \multirow[t]{2}{*}{ Constante } & 0.002859 & 0.00307 & 0.002957 & 0.002599 \\
\hline & $(0.00221)$ & $(0.0022)$ & $(0.00224)$ & $(0.00205)$ \\
\hline \multicolumn{5}{|l|}{ Equação da variância } \\
\hline \multirow[t]{2}{*}{ Constante } & $0.000128^{*}$ & $0.000089^{*}$ & $0.000137^{*}$ & $0.000474^{*}$ \\
\hline & $(0.00003)$ & (0000026) & $(0.00004)$ & $(0.00013)$ \\
\hline \multirow[t]{2}{*}{ ARCH (1) } & $0.067045^{*}$ & $0.082989^{*}$ & $0.076199 *$ & $0.094547^{*}$ \\
\hline & $(0.01591)$ & $(0.02678)$ & $(0.0269)$ & $(0.01773)$ \\
\hline \multirow[t]{2}{*}{$\mathrm{ARCH}(2)$} & - & -0.032656 & - & $0.07264^{*}$ \\
\hline & - & $(0.02382)$ & - & $(0.0166)$ \\
\hline \multirow[t]{2}{*}{ GARCH (1) } & $0.898325^{*}$ & $0.925976^{*}$ & $0.648784^{*}$ & $-0.147096^{*}$ \\
\hline & $(0.01906)$ & $(0.01527)$ & $(0.36027)$ & $(0.04070)$ \\
\hline \multirow[t]{2}{*}{ GARCH (2) } & - & - & 0.239276 & $0.813449^{*}$ \\
\hline & - & - & $(0.33335)$ & $(0.03677)$ \\
\hline \multirow[t]{2}{*}{ Assimetria } & $-0.0293^{* * *}$ & -0.020505 & -0.033045 & $-0.053722^{*}$ \\
\hline & $(0.01717)$ & $(0.01415)$ & $(0.02066)$ & $(0.01576)$ \\
\hline AIC & -3.279 & -3.277 & -3.276 & -3.291 \\
\hline SBC & -3.236 & -3.225 & -3.224 & -3.230 \\
\hline Log. Verossimilhança & 803.636 & 804.025 & 803.731 & 808.371 \\
\hline
\end{tabular}

*Significativo ao nível 1\%; ** Significativo ao nível de 5\% ; ** Significativo ao nível de 10\% pelo teste z; + Modelo selecionado. Desvios padrão entre parênteses. Fonte: Resultados da pesquisa. 
sistemáticas, causando incerteza para parte dos produtores familiares. A aplicação de políticas no mercado de mamona, como o PNPB, bem como a entrada e a saída de agentes que atuam nesse setor, podem aumentar a volatilidade desse mercado por um longo período. Tais eventos, caracterizados como choques, teriam efeitos de aumentar a volatilidade do preço da mamona, elevar o risco de preço e tornar a atividade menos atrativa para a agricultura familiar.

A persistência dos choques e a volatilidade do mercado não seriam um grande problema para as usinas de biodiesel, uma vez que os mercados de matérias-primas alternativas também apresentam tal comportamento, como o da soja no mercado brasileiro (SAITH e KAMITANI, 2012; SILVA, SÁFADI e CASTRO JÚNIOR, 2005) e do óleo de dendê no mercado internacional (GONTIJO, FERNANDES e SARAIVA, 2011). A volatilidade estrutural, por seu turno, afeta a decisão dos produtores rurais, uma vez que caracteriza a atividade como arriscada em relação a preços.
Adicionalmente, verificou-se que elevação nos preços aumenta a volatilidade, impactando no risco de mercado. O coeficiente de assimetria do modelo TGARCH $(2,2)$, de -0.053722 , significativo, indica que inovações positivas (aumentos de preço) provocam maior instabilidade nos preços da mamona que inovações negativas (quedas de preços) (Tabela 5). A estimação por EGARCH $(2,2)$ também indicou tal assimetria, constatada pelo coeficiente de assimetria na Tabela 6. Tais resultados indicam que um choque positivo é mais persistente que um choque negativo. Assim, choques positivos podem elevar o retorno médio esperado, porém, também aumentam a variância, provocando aumento de risco da atividade. Considerando que no contexto do PNPB os preços da mamona se elevaram, conforme indicaram Diniz e Favareto (2012) e Gonçalves, Favareto e Abromovay (2013), essa elevação levou a uma maior volatilidade e risco de preço à atividade. Dessa forma, as políticas que busquem estimular a entrada de agricultores no mercado de mamona via elevação dos preços pagos estariam associa-

Tabela 6. Comparação de desempenho entre os modelos EGARCH de volatilidade

\begin{tabular}{|c|c|c|c|c|}
\hline Retornos Mamona & EGARCH $(1,1)$ & EGARCH $(2,1)$ & EGARCH $(1,2)$ & EGARCH $(2,2)$ \\
\hline Equação da média & & & & + \\
\hline \multirow[t]{2}{*}{ Constante } & 0.002974 & 0.003222 & 0.003071 & 0.00274 \\
\hline & $(0.00209)$ & $(0.00213)$ & $(0.00212)$ & $(0.0018)$ \\
\hline \multicolumn{5}{|l|}{ Equação da variância } \\
\hline \multirow[t]{2}{*}{ Constante } & $-0.277076^{*}$ & $-0.266788^{*}$ & $-0.320129^{*}$ & $-1.654854^{*}$ \\
\hline & $(0.05812)$ & $(0.05658)$ & $(0.13961)$ & $(0.24030)$ \\
\hline \multirow[t]{2}{*}{ absARCH (1) } & $0.069631^{*}$ & $0.097492^{*}$ & $0.081127^{*}$ & $0.16849^{*}$ \\
\hline & $(0.01246)$ & $(0.04192)$ & $(0.03928)$ & $(0.0235)$ \\
\hline \multirow[t]{2}{*}{ absARCH (2) } & - & -0.030467 & - & $0.094599^{*}$ \\
\hline & - & $(0.03918)$ & - & $(0.02677)$ \\
\hline \multirow[t]{2}{*}{ lnGARCH (1) } & $0.960499 *$ & $0.961963^{*}$ & 0.774907 & $-0.126091^{*}$ \\
\hline & $(0.00935)$ & $(0.00911)$ & $(0.50524)$ & $(0.01525)$ \\
\hline \multirow[t]{2}{*}{$\operatorname{lnGARCH}(2)$} & - & - & 0.179511 & $0.883247^{*}$ \\
\hline & - & - & $(0.48813)$ & $(0.02203)$ \\
\hline \multirow[t]{2}{*}{ Assimetria } & 0.007187 & 0.007084 & 0.008637 & 0.02128 \\
\hline & $(0.01121)$ & $(0.01117)$ & $(0.01324)$ & $(0.0179)$ \\
\hline AIC & -3.282 & -3.278 & -3.278 & -3.344 \\
\hline SBC & -3.239 & -3.227 & -3.226 & -3.284 \\
\hline Log. Verossimilhança & 804.262 & 804.346 & 804.3015 & 821.388 \\
\hline
\end{tabular}

*Significativo ao nível 1\%; ** Significativo ao nível de 5\%; ***Significativo ao nível de $10 \%$ pelo teste z; + Modelo selecionado. Desvios padrão entre parênteses.

Fonte: Resultados da pesquisa. 
das a uma maior volatilidade, aumentando a incerteza de preços e, talvez, desestimulando a participação de agricultores avessos ao risco.

A Tabela 7 apresenta resultados para a estimativa da variável dicotômica para o período pós-PNPB gerada pelos modelos TGARCH e EGARCH. Em ambos os modelos, a variável mostrou-se relevante, tendo o período pós-PNPB apresentado maior nível de volatilidade que o período anterior. Tal relação indica que os diversos eventos que ocorreram ao longo do PNPB citados por César e Batalha (2010a, 2010b, 2011), tais como entrada e saída de agentes, políticas de incentivo, criação de um novo mercado para a mamona e choques em mercados de outras oleaginosas elevaram a volatilidade desse mercado.

A Figura 5 traz as estimativas da volatilidade dos retornos dos preços da mamona obtidos pelos modelos TGARCH e EGARCH. Os resultados confirmam os das variáveis categóricas. É perceptível que existe maior nível de volatilidade a partir de 2004, coincidindo com o lançamento do PNPB.
A maior instabilidade dos preços se associa ao maior risco referente à atividade, principalmente no cenário envolvendo pequenos produtores. $\mathrm{O}$ fato de a mamona estar sendo adquirida pelo PNPB para atender à exigência do selo social pode estar influenciando na relação entre o PNPB e o período com maior volatilidade nos preços da mamona. Se por um lado isso assegura demanda para os pequenos produtores de mamona, por outro, gera maior instabilidade/risco para o mercado e desestimula sua produção.

Em outras palavras, o programa criado pelo governo para minimizar o risco alimentar e a pobreza rural do Nordeste via adoção da mamona como cultura estratégica pode se tornar inviável pelos mecanismos do próprio programa. De fato, os resultados encontrados neste trabalho trazem uma dimensão quantitativa da instabilidade que ocorreu no setor após a implantação do PNPB, conforme verificado por trabalhos com enfoques qualitativos que analisaram o setor (CÉSAR e BATALHA, 2010a, 2010b, 2011; WATANABE, BIJMAN e SLINGERLAND, 2012).

Tabela 7. Estimativas da variável categórica para o período pós PNPB

\begin{tabular}{lcccc}
\hline \multicolumn{1}{c}{ Estimativa } & Coeficiente (PNPB) & Desvio padrão & Estatística z & Significância \\
\hline TGARCH $(2,2)$ & 0.0004 & 0.000133 & 3.012255 & 0.0026 \\
EGARCH $(2,2)$ & 0.1883 & 0.067811 & 2.777742 & 0.0055 \\
\hline
\end{tabular}

Fonte: Resultados da pesquisa.

Figura 5. Volatilidade estimada dos preços semanais de mamona pelos modelos TGARCH $(2,2)$ (esquerda) e EGARCH $(2,2)$ (direita)
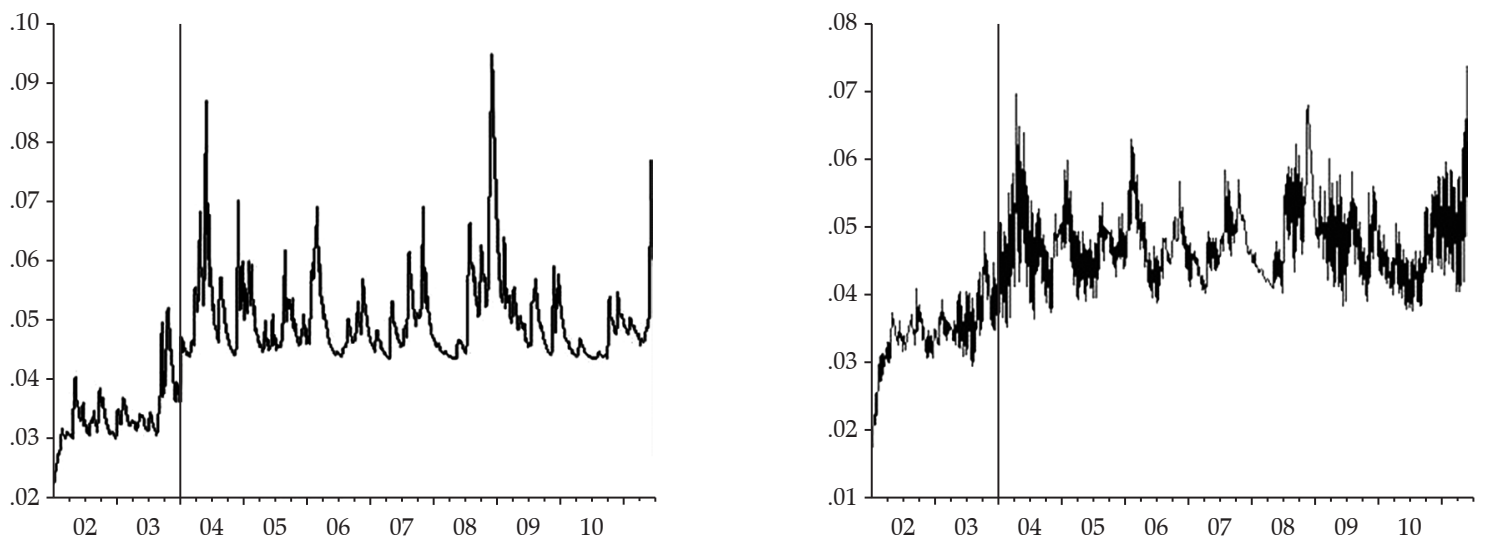

Fonte: Resultados da pesquisa. 


\section{Considerações finais}

A cultura da mamona foi adotada como estratégica no contexto do PNPB pela sua potencialidade para reduzir a pobreza rural, principalmente no Nordeste. A princípio, a ideia era adquirir mamona para introduzi-la na matriz energética mais sustentável proposta pelo programa. No entanto, atualmente, sua participação para o biodiesel é nula. O que se observou na última década na Bahia, maior produtor nacional, foi uma forte instabilidade tanto na produção quanto no preço da mamona. Esse fato pode estar ligado às questões de aversão ao risco e segurança alimentar dos pequenos produtores, foco do programa.

Foram constatadas persistência e assimetria quanto às inovações no preço, o que torna o mercado da mamona mais volátil e deixa a atividade menos atrativa para o pequeno produtor devido sua aversão ao risco. Assim, qualquer mudança nesse mercado por consequência de aplicação de políticas públicas pode não levar a um aumento da produção e da inserção da agricultura familiar nesse mercado.

Algumas questões ainda não ficaram claras neste trabalho, podendo ser tratadas em trabalhos futuros. Entre as mais importantes, estariam a análise do trade-off entre risco e rentabilidade por parte dos produtores para quantificar efetivamente o impacto do aumento da volatilidade sobre a produção de mamona. Essa abordagem necessitaria de forte embasamento na teoria microeconômica relacionada à escolha sob incerteza e permitiria verificar se políticas de preço, distribuição de alimentos, adoção de tecnologia e de crédito rural poderiam impactar na propensão de o produtor familiar produzir ou não mamona em um contexto de maior risco de preço. Outra questão relevante é a relação entre os preços da mamona e outras matérias-primas utilizadas na produção de biodiesel. Por abrir um novo mercado para a mamona, o PNPB também adicionou novas fontes de instabilidade de preço para esse mercado, uma vez que a mamona tem inúmeros substitutos para a produção de biodiesel.

\section{Referências}

ANP - AGÊNCIA NACIONAL DE PETRÓLEO. Boletim mensal do biodiesel: Agosto de 2015. Disponível em: </l www.anp.gov.br/?pg $=63697 \& \mathrm{~m}=\& \mathrm{t} 1=\& \mathrm{t} 2=\& \mathrm{t} 3=\& \mathrm{t}$ $4=\&$ ar $=\& p s=\&$ cachebust $=1362160670579>$. Acesso em: 28 set. 2015.

BOLLERSLEV, T. Generalized autoregressive conditional heteroskedasticity. Journal of Econometrics, v. 31, p. 307327, 1986.

BREUSCH, T. S. e GODFREY, L. G. A review of recent work on testing for autocorrelation in dynamic simultaneous models. In: CURRIE, D., NOBAY, R. e PEEL, D. (Eds.). Macroeconomic Analysis, Essays in Macroeconomics and Economics. 1. ed. London: Croom Helm, 1981. p. 63-100.

BUENO, R. L. S. Econometria de Séries Temporais. 1. ed. São Paulo: Cengage Learning, 2008.

CAMPOS, M. B. N. et al. Análise da competitividade da produção de oleaginosas oriundas da agricultura familiar na região de abrangência da Coopaf. Bahia Análise \& Dados, v. 18, n. 4, p. 687-697, 2009.

CÉSAR, A. S. e BATALHA, M. O. Biodiesel in Brazil: History and relevant policies. African Journal of Agricultural Research, v. 5, n. 11, p. 1147-1153, 2010a.

. O. Biodiesel production from castor oil in Brazil: A difficult reality. Energy Policy, v. 38, n. 8, p. 4031-4039, ago. 2010b.

. Análise dos direcionadores de competitividade sobre a cadeia produtiva de biodiesel: o caso da mamona. Produção, v. 21, n. 3, p. 484-497, set. 2011.

CONAB - COMPANHIA NACIONAL DE ABASTECIMENTO. Indicadores. Disponívelem: $<$ http:// www.conab.gov.br/conteudos.php? $\mathrm{a}=1382 \& \mathrm{t}=2>$. Acesso em: 1 fev. 2013.

DICKEY, D. A. e FULLER, W. A. Distribution of the estimators for autoregressive time series with unit root. Journal of the American Statistical Association, v. 74, n. 366, p. 427-431, 1979.

. Likelihood ratio statistics for autoregressive time series with a unit root. Econometrica, v. 49, n. 4, p. 1052-1072, 1981.

DINIZ, J. F. e FAVARETO, A. Os desafios da inclusão da agricultura familiar no mercado de matéria-prima para o biodiesel no Brasil. Estudos Sociedade e Agricultura, v. 20, n. 1, p. 139-187, 2012. 
ENGLE, R. F. Autoregressive conditional heteroscedasticity with estimates of the variance of United Kingdom inflation. Econometrica, v. 50, n. 4, p. 987-1007, 1982.

\section{FAO - FOOD AND AGRICULTURE ORGANIZATION} OF THE UNITED NATIONS. Food price index. Disponível em: <//www.fao.org/worldfoodsituation/ wfs-home/en >. Acesso em: 10 out. 2012.

GLOSTEN, L. R., JAGANATHAN, R. e RUNKLE, D. E. On the relation between the expected value and the volatility of the nominal excess return on stocks. The Journal of Finance, v. 48, n. 5, p. 1779-1801, 1993.

GONÇALVES, Y. K., FAVARETO, A. e ABRAMOVAY, R. Estruturas sociais no semiárido e o mercado de biodiesel. Caderno CRH, v. 26, n. 68, p. 347-362, 2013.

GONTIJO, T. S., FERNANDES, E. A. e SARAIVA, M. B. Análise da volatilidade do retorno da commodity dendê: 1980-2008. Revista de Economia e Sociologia Rural, v. 49, n. 4, p. 857-874, dez. 2011.

IBGE - INSTITUTO BRASILEIRO DE GEOGRAFIA E ESTATÍSTICA. Pesquisa Agrícola Municipal.

MENDES, A. P. A. e COSTA, R. C. Mercado brasileiro de biodiesel e perspectivas futuras. BNDES Setorial, v. 31, p. 253-280, 2010.

NELSON, D. B. Conditional heteroskedasticity in asset returns: a new approach. Econometrica, v. 59, n. 2, p. 347-370, 1991.

OTUKI, T. F., WEYDMANN, C. L. e SEABRA, F. Febre aftosa e volatilidade dos preços do produtor de carne suína. Revista de Economia e Agronegócio, v. 7, n. 2, p. 235258, 2009.

SAITH, W. e KAMITANI, E. L. T. Volatilidade e assimetria de choques no mercado agropecuário brasileiro um uso dos modelos da família GARCH. Tecnologia \& Ciência Agropecuária, v. 6, n. 2012, p. 1-7, 2012.
SCHOLZ, V. e SILVA, J. N. Prospects and risks of the use of castor oil as a fuel. Biomass and Bioenergy, v. 32, n. 2, p. 95-100, fev. 2008.

SEAGRI. Cotações Agrícolas. Disponível em: <http://www. seagri.ba.gov.br/cotacao.asp.>. Acesso em: 1 fev. 2013.

SILVA, M. S. et al. Family farming and biodiesel: The difficulties of socioeconomic inclusion in the Northeast of Brazil. Journal of Agricultural Science, v. 6, n. 7, p. 231252, 2014a.

. Uma análise institucional no quadro regulatório do biodiesel no Brasil à luz da teoria dos custos de transação. Revista de Desenvolvimento Econômico, v. 16, n. 29 , p. 25-38, 2014b.

SILVA, W. S., SÁFADI, T. e CASTRO JÚNIOR, L. G. Uma análise empírica da volatilidade do retorno de commodities agrícolas utilizando modelos ARCH: os casos do café e da soja. Revista de Economia e Sociologia Rural, v. 43, n. 1, p. 119-134, mar. 2005.

TAYLOR, S. J. Modelling Financial Time Series. 1. ed. Chichester: JohnWiley \& Sons Ltd, 1986.

TODARO, M. P. e SMITH, S. C. Economic Development. 11. ed. Boston: Addison-Wesley, Pearson, 2012.

VIANA, K. R. O., PEREZ, R. e MACHADO, C. T. C. Risco do cultivo da mamona promovida pelo programa do biodiesel brasileiro. Revista Agrotecnologia, v. 2, n. 2, p. 15-29, 2011.

WATANABE, K., BIJMAN, J. e SLINGERLAND, M. Institutional arrangements in the emerging biodiesel industry: case studies from Minas Gerais - Brazil. Energy Policy, v. 40, p. 381-389, jan. 2012.

WHITLE, P. Hypothesis testing in time series analysis. Vol. 4. ed. [s.1.] Almqvist \& Wiksells, 1951.

ZAKOIAN, J. Threshold heteroskedastic models. Journal of Economic Dynamics and Control, v. 18, p. 931955, 1994. 